\title{
First direct 3D visualisation of microstructural evolutions during sintering through X-ray computed microtomography
}

\section{Dominique Bernard, Damien Gendron, J ean-Marc Heintz, Sylvie Bordère , J ean Etourneau}

\begin{abstract}
:
X-ray computed microtomography (XCMT) has been applied to ceramic samples of different materials to visualise, for the first time at this scale, real 3D microstructural evolutions during sintering. Using this technique, it has been possible to follow the whole sintering process of the same grains set. Two materials have been studied; a glass powder heat treated at $700 \mathrm{C}$ and a crystallised lithium borate ( Li6Gd(BO3)3) powder heat treated at $720 \mathrm{C}$. XCMT measurements have been done after different sintering times. For each material, a sub-volume was individualised and localised on the successive recordings and its 3D images numerically reconstructed. Description of the three-dimensional microstructures evolution is proposed. From the 3D experimental data, quantitative evolutions of parameters such as porosity and neck size are presented for the glass sample. Possibilities offered by this technique to study complex sintering processes, as for lithium borate, are illustrated.
\end{abstract}

Keywords : Sintering; Microstructure; Non-destructive testing; Grain interface; X-ray computed microtomography

\section{Introduction}

Sintering is the material preparation process that leads to consolidation through particle bonding and porosity elimination at temperatures lower than their melting point. During the heat treatment, mass transfer takes place to minimise surface energies and large microstructural changes occur, leading to densification and grain growth.

Since the sixties, many works have been done to describe and model the sintering mechanisms and processes. The first attempts considered sintering from a phenomenological point of view [1-5]. For ceramics, the complete process was roughly divided in three steps corresponding respectively to neck growth, pore elimination and final densification with grain growth. Experimental data that are used in these models rely on large-scale techniques (dilatometry, specific area measurements) or average data obtained from bi-dimensional measurements (grain size and pore size determined on sections by image analysis).

Such approaches gave a good understanding of the macroscopic behaviour of powders, especially in the case of glass powders $[6,7]$. However, it remains insufficient to predict precisely densification phenomena in more general cases where non-ideal arrangements of grains are present $[8,9]$. On the other hand, improvements in sintering modelling have been achieved in the past years taking into account agglomeration effects as well as particle-particle contact shapes and pore geometries [916]. In other words, sintering is now considered at the grain scale and major advances are expected to come from numerical models that can handle such micro-geometries all along the sintering process. Such theoretical efforts need experimental data to sustain the models and to validate their assumptions. While description of macroscopic behaviour is quite easy to obtain from the previous mentioned techniques (even if these techniques are generally only pertinent for one of the three 
sintering stages), there is actually a lack of direct and volumic (3D) microstructure characterisation techniques that can be used during sintering and at the micron scale.

In fact, X-ray tomography could fit quite well these requirements. It is a technique that has been originally developed for medical imaging, taking advantage of inhomogeneous absorption of X-rays in bodies. Now, due to the huge progresses that have been made in resolution (micron scale measurements are conceivable) and in 3D visualisation, X-ray computed microtomography (XCMT) becomes a very interesting tool for material sciences $[17,18]$ and especially for ceramic processing.

Some interesting results have already been presented concerning porosity characterisation of compacted powders, although measurement remained at low resolution and the 3D reconstructions have not been presented [19-21]. The present work actually refers to that technique to propose a 3D characterisation of two compacts during sintering. In this paper are reported the experiments [22] that have been carried out on a glass powder sample and a lithium borate sample during their sintering and, for the first time, 3D microstructure evolutions are shown at a high resolution, i.e., $2 \mu \mathrm{m}$.

\section{Experimental procedures}

Sintering mechanism understanding being the main objective of this work, we looked for materials available under the form of regular grains with a size range (between 100 and $200 \mu \mathrm{m}$ ) allowing to have a significant number of grains under the X-ray beam while a high voxel resolution was kept. The first selected material was a commercial soda-lime glass powder (Sovitec, Glaverbel SA, softening temperature: $730{ }^{\circ} \mathrm{C}$ ) representing an ideal and widely studied system for viscous sintering. The second material has been selected to study sintering of crystallised particles. The different specifications that were imposed for the experiments (grains diameter, sintering temperature and sintering time) excluded the most classical ceramic materials and guided us towards a more unusual one easily prepared at the ICMCB, i.e., the lithium borate.

Specimens of soda-lime glass powder were prepared as followed. The powder was compacted under uniaxial pressure ( $1.5 \mathrm{MPa}$ ) to form cylinders ( $3 \mathrm{~mm}$ in diameter and $5 \mathrm{~mm}$ in height). Polyvinylalcool (PVA, $1 \mathrm{wt} \%$ ) was used as a binder to promote mechanical strength in the green state. A first heat treatment, at $650{ }^{\circ} \mathrm{C}$ for $1 \mathrm{~h}$, was done to burn out the PVA $\left(2.5^{\circ} \mathrm{C} \mathrm{min}^{-1}\right.$ heating rate). A second heat treatment was done at $700{ }^{\circ} \mathrm{C}$ during $10 \mathrm{~min}$ to pre-sinter the samples in order to make it strong enough for the subsequent isothermal sintering experiments. In fact, these as-obtained samples, very poorly densified, were considered as the starting point to study the microstructure evolution. Isothermal sintering was realised by introducing directly the sample in a furnace, hold at $700{ }^{\circ} \mathrm{C}$. To prepare lithium borate powder samples, first, massive crystals grown at ICMCB to develop new neutron detectors [23] were ground to obtain a fine powder. A uniform layer of this powder was deposited on a vitreous carbon plate on which several $100 \mu \mathrm{m}$ width channels were grooved. Then, the plate and the powder were placed in a furnace at $870{ }^{\circ} \mathrm{C}$ melting temperature of lithium borate) during $10 \mathrm{~h}$ under an argon-hydrogen atmosphere to limit carbon oxidation. The lithium borate slowly melted and, as it poorly wets the vitreous carbon, small droplets formed with diameters close to the channels width. During the subsequent cooling the lithium borate droplets crystallised, keeping their spherical shape. At room temperature, the obtained spherical crystals were sieved to fit the desired diameters distribution.

$X$-rays diffraction measurements were performed to check the crystallised state of the resulting material that actually corresponded to $\mathrm{Li6Gd(BO3)3.} \mathrm{As} \mathrm{a} \mathrm{matter} \mathrm{of} \mathrm{fact} \mathrm{this} \mathrm{material} \mathrm{undergoes} \mathrm{weight}$ losses when heated above $700{ }^{\circ} \mathrm{C}$. Therefore a sintering temperature of $720^{\circ} \mathrm{C}$ was chosen to allow sintering phenomena to take place with a limited evaporation of lithium borate (a loss of $7 \%$ was observed after a $1 \mathrm{~h}$ heat treatment at $720^{\circ} \mathrm{C}$ ). As for glass powder, a pre-sintering, at $720^{\circ} \mathrm{C}$ during $10 \mathrm{~min}$, was realised to be sure that the samples could be manipulated. The different sintering steps were done at $720^{\circ} \mathrm{C}$ at the European Synchrotron Radiation Facility (ESRF, Grenoble, France) site and 
the maximum duration of sintering treatment was set to $190 \mathrm{~min}$. XRD of the sintered material only revealed $\mathrm{Li}_{6} \mathrm{Gd}\left(\mathrm{BO}_{3}\right)_{3}$ phase. However, the observed weight loss as well as the modification of the grains surface appearance suggested that the mechanisms involved in this experiment were not as simple as expected. X-ray computed microtomography is the up to date development of X-ray tomography, which consists in detecting the residual energy of a beam that passes through a sample [24]. The specimen is rotated under the X-ray beam and a large number of radiographs (projections) are recorded at different angles on the detector plane. Some reference images are added to control the noise and the homogeneity of the incident beam. The 3D representation of the X-ray absorption by the sample is then numerically reconstructed from all these $2 \mathrm{D}$ images. The most commonly used algorithm for reconstruction is the filtered back-projection method [25-27]. Different beam sources are available for tomography experiments. In the present work, we used the synchrotron radiation from the ESRF (high intensity source, from 106 to 1012 times the usual X-ray ones, low angle emission, large and continuous emission spectrum). Resolutions are ranging from 40 to $1 \mu \mathrm{m}$. Recent developments realised at ESRF allow to reach a $0.3 \mu \mathrm{m}$ resolution which justifies the "micro" denomination for that type of X-ray tomography. I sothermal sintering treatments and microtomography measurements were sequentially performed on the pre-sintered samples at ESRF on the ID19 imaging and diffraction beamline. The used energy was $17.5 \mathrm{keV}$, which was adapted to the nature and the thickness of the material. A typical experiment consisted in recording 900 projection images and about 100 reference images for flat field correction. The final image is reconstructed using sequences of filtered back projection algorithm run on each slice of the volume after rearranging the data in a set of corrected sinograms [17]. This technique remains quite heavy to handle, especially considering the huge amount of data to be treated for volume reconstruction (from 2 to 13 Goctets per tomography depending of the detector). The spatial resolution mainly depends on the optic system that determines the effective pixel size on the detector. During these experiments, a $2 \mu \mathrm{m}$ resolution was used. However, some limitations related to the size of the back detector occurred. At ESRF, the diameter actually scanned was 1024 times the chosen resolution. Considering our sample diameter $(3 \mathrm{~mm})$ and the used resolution $(2 \mathrm{Im})$, the samples were not completely in the CCD camera field, which corresponds to what is called local tomography. The sample part that was actually scanned during the XCMT experiments corresponded to cylinders of about $1.9 \mathrm{~mm}$ in diameter and $0.8 \mathrm{~mm}$ in height. This procedure could induce some artefacts [17] but, in our case, no noticeable degradation of the data quality was observed.

\section{Results}

The first results obtained from the measurements performed on the soda-lime glass sample concern the visualisation of 2D sections through the scanned volume of the sample. Different sections were chosen at approximately the same height within the sample to illustrate the material evolution as a function of the sintering time (Fig. 1). These images are only 2D views. However, they concern the same sample and the same area, and as such they provide interesting and original insights on the changes associated to sintering. For short sintering times, ts $=20 \mathrm{~min}$, grain sections appear as almost perfect disks. Grain arrangement remains close to the initial configuration that corresponds to a dense random packing of spheres ( $36 \%$ porosity calculated from the section).

Very few defects, like broken spheres due to the compression or grain-pairs formed during the reparation of the glass particles, are present in the system. For that short sintering time, the densification process was at its very beginning and the $2 \mathrm{D}$ representation given by that section (Fig. $1(\mathrm{a})$ ) only reveals a few necks between particles. For intermediate sintering time, ts $=120 \mathrm{~min}$, densification has clearly begun and the porosity is now about $27 \%$. Grains can still be considered as spherical but now most of them are connected to each other. For ts $=180 \mathrm{~min}$, densification proceeded and porosity has decreased down to $16 \%$. Grains no more appear as spherical and it 
becomes difficult to view them as individual entities. For the longer sintering time, ts $=270 \mathrm{~min}$, only the larger pores remain and the overall porosity has gone down to $8 \%$. At this stage, microstructural evolutions became weak, illustrating the slowing down of the sintering kinetics. These views illustrate well the three stages of sintering. But very few quantitative and relevant morphological measurements, number of contacts or neck size for example, can be done on such 2D images. It will not be the case in 3D. Squares superimposed on the previous images of sections (Fig. 1) show the area that were numerically reconstructed in order to follow the 3D evolution of this glass material during heat treatment. Small internal bubbles were used as markers to insure that the same set of particles was considered. The size of the reconstructed sub-volume was $200 \cdot 200 \cdot 200 \mathrm{Im3}$. The 3D microstructural evolution during sintering of the same sub-volume of the compact, extracted from the whole sample, is presented in Fig. 2, for the solid part and the void part, left and right columns, respectively. From these images, one can easily visualise necks between particles and, according to the sintering time, their growth. Also, formation of new necks can be identified, as well as disruption of "ligament" pores at the final sintering stage. Fig. 2(a) shows the compact after 20 min of sintering, in which some small size necks are already present. As sintering is proceeding (Fig. 2(b): ts $=120 \mathrm{~min}$ ), grain arrangement slightly changes and formation of new necks between particles can be observed (see arrows) as well as in increase in size of the previous ones. For longer sintering times, neck growth and changes in grain shape become important. For the last illustrated sintering time, (Fig. 2(d): $t=270 \mathrm{~min}$ ), the solid phase almost fills the volume of the reference cube and the grains no longer appear as spherical in shape. The right column of Fig. 2 presents the dual part of the reconstruction, which confirms the previous observations and gives also complementary information. As a matter of fact, evolution of necks between grains appears even more clearly on these views. The formation of a new neck that can be suspected on the solid part in Fig. 2(b), is actually confirmed by the presence of a hole in the pore space between these two grains, which did not exist in the previous view (Fig. 2(a)). Also, images of the porosity clearly illustrate its complex geometry all along the sintering process. It shows that the classical geometrical model used for the intermediate stage, where pores are considered as intersecting cylinders [5], oversimplify the reality. Indeed, one can notice that pore channels tend to collapse for the longest sintering times and conditions of pore separation, as observed in the last picture (Fig. 2(d)), could then be studied using these data. The same procedure has been followed for the lithium borate sample. Three images of the same section at different sintering times are presented on Fig. 3 . The evolution illustrated here differs greatly from the one pictured on Fig. 1 although the initial states were quite similar: porosity is about the same $(36 \%)$, grains present rather regular forms and few contacts between them are formed. The major difference at this stage was the presence of heterogeneities within the grains of lithium borate (in grey on Fig. 3 ) in a small proportion (about $0.6 \%$ of the volume at ts $=10 \mathrm{~min}$ ). This parasitic phase appeared in white (material more opaque to X-rays) on Fig. 3 either in the form of crystallites (Fig. 3(a), arrow A) or in the form of platelets (Fig. 3(a), arrow B). During sintering, a limited densification occurred (at ts $=100$ min porosity was equal to $29 \%$ and to $26 \%$ at ts $=190 \mathrm{~min})$ and the volume fraction of the unknown phase slightly increased $(0.8 \%$ at ts $=$ $100 \mathrm{~min}$ and $1 \%$ at ts $=190 \mathrm{~min})$. Necks that appeared at the contacts between grains present specific configuration (Fig. 3(c)). Such a morphology is usually associated with the initial stage of a liquid-phase sintering [9]. The presence of a liquid phase generally facilitates the densification, but if another mechanism, like evaporation/condensation, is also operative, this can strongly limit the final density. As a matter of fact, the modification of the aspects of the grains surface and the weight loss noticed along the heat treatment (about $7 \%$ per hour at $720^{\circ} \mathrm{C}$ ) corresponded likely to an vaporation/condensation mechanism. Both analyses are strong arguments in favour of such a combination of mechanisms. 3D visualisations (Fig. 4) of the solid phases, of the void space and of the unknown solid phase confirmed the inferences made from the 2D observations: there are several mechanisms simultaneously involved in the sintering of the lithium borate. Fig. 4 shows that with synchrotron microtomography it is possible to obtain precise information on the different phases that are present as long as their coefficients of absorption are distinct and this, even 
when the volume fraction of one phase is too small to permit its detection by XRD. However, in the present case, some complementary studies are necessary to confirm the presence of a liquid phase (direct observation of the sintering of a few grains under a microscope) and to precisely determine the composition of the material in order to design a microtomography experiment that could produce quantitative information on the sintering process itself.

\section{Discussion}

As already mentioned, some relevant parameters relative to sintering phenomena can be extracted from the images of the soda lime glass sample. The first one deals with the calculation of the porosity both in the whole sample and in the small studied volume. Due to the high quality of the recordings, well-defined 2D grey scale slice images were obtained (Fig. 1). Taking advantage of that contrast, relative proportion of the porous phase to the solid phase was easy to calculate on each slice. The whole porosity of the compact was then obtained from cumulative measurements. The same image analysis procedure was realised on the small 3D images (Fig. 2). Comparison of these two porosity values as a function of sintering time is proposed in Fig. 5 . The same trend is observed whatever the investigated volume and the agreement between the two measurements is good. It shows that the small chosen volume can be used to follow the pore elimination process during densification of the glass powder. The other interesting point concerns the different scales of analysis accessible using the XCMT technique. Porosity measurements on the whole sample by XCMT bring the same information than dilatometry or mercury porosimetry experiments. Conversely, determination of the porosity by XCMT both at the macroscopic and at a reduced scale allows for example, the influence of large packing defects on the surrounding particles during densification to be studied. These investigations are of special interest in order to better describe sintering of real materials containing many defects in the green state. The second information that was derived from these images deals with the evolution of necks between particles. The neck parameter is still largely used to determine preponderant sintering mechanism [9]. Determination of the size of the neck is a more complicated operation, which needs to manipulate the image in space. This is feasible in the case of the glass power sample owing to the high quality of the 3D images. On Fig. 6 are superimposed three images of the same grain at ts $=20 \mathrm{~min}$ (red lines), ts $=90 \mathrm{~min}$ (transparent blue) and ts $=120 \mathrm{~min}$ (yellow) were the evolution of one neck is directly visualised. Moving the volume up to the point where neck plane coincides with the measurement grid plane allows to measure its dimensions. Four necks were analysed by this method as a function of the sintering time (Fig. 7). Three of them were present from the beginning of sintering (ts $=20 \mathrm{~min}$ ) while the last one (marked 4 ) appeared lately (ts $=90 \mathrm{~min}$ ). Two perpendicular diameters were measured for each neck (expressed as $X$ and $Y$ in Fig. 7). One can notice that the increase in size of the three first necks follows the same kinetics. Moreover, the fourth one also behaves in the same way, only shifted in time. This suggests that neck growth can actually be represented by a unique behaviour within that glass material. In fact, we have shown by numerical calculation (Monte-Carlo method) on different grain configurations (line, arrays) that the increase in size of neck growth at the beginning of the sintering process of crystalline and glass materials follows a similar kinetics than the evolution of the dihedral angle toward its equilibrium value [28]. It corresponds mainly to surface energy minimisation in the vicinity of the neck and therefore it is insensitive to long scale ordering of grains or to their packing arrangement. The observed neck growth behaviour, similar for the four studied necks, seems then in agreement with these numerical calculations, which means that during the first stage of glass material sintering, neck growth might be almost independent of the grain surrounding. Moreover, we have shown elsewhere [29] that this experimental neck growth kinetics does not follow the classical t $1 / 2$ law (Frenkel_s model) related to viscous flow. The fraction $1 / 2$ is an exponent for $t$. In fact, sintering of the studied glass in that temperature range is driven by a viscoelastic sintering mechanism like the one that can be observed for polymer particle sintering. 
Now, these preliminary measurements should be completed (determination of localised porosity as a function of defects, co-ordination number evolution in time, etc.) and extended to the whole sample. These 3D experimental data will be compared to evolutions predicted by viscous like sintering models.

\section{Conclusions}

For the first time, direct 3D visualisation of microstructural evolutions during isothermal sintering have been achieved using $X$-ray computed microtomography. This demonstrates the huge potentiality of this non-invasive and non-destructive technique for the analysis of sintering phenomena. In such an ideal system like the soda-lime glass sample, the different sintering stages were clearly identified. Some preliminary quantitative measurements have also been performed on the 3D images.

Porosity evolution has been followed both at the scale of the whole sample and in a small sub-volume. The (200) $3 \mathrm{Im} 3$ analysed sub-volume has given a good estimation of the densification process. Neck growth has been determined and a unique neck growth behaviour seems to be operative for that "monodispersed" system. For more complex systems like the lithium borate sample, for which several different mechanisms are acting simultaneously, possibilities can even be greater using specific capabilities of third generation synchrotron facilities [17]: easy and precise energy tuning, allowing the use of different energies for scanning the same sample, and coherence of the beam, permitting 3D imaging by holotomography (also called phase contrast tomography). The use of those sophisticated methods to analyse individually the different mechanisms involved requires a complete preliminary characterisation of the material.

3D imaging by microtomography is a mature technique but a lot of work has still to be done to really exploit all its potentialities. Optimal treatments and efficient methods of analysis of the huge amount of 3D data generated (several Giga Bytes got each scan) are currently searched for by different groups in the world including ours in ICMCB. Different algorithms developed for medical imaging (3D registration, combination of images from different sources, etc.), might be adapted to material science in order to obtain statistical results and to extract experimental data that will "feed" modelling and sintering simulations.

\section{Acknowledgements :}

The authors thank Elodie Boller and Jose' Baruchel (ID 19, ESRF, Grenoble) for the scientific support concerning tomography experiments. Computations and visualisations have been made possible thanks to partial funding of the "Conseil Re' gional d_Aquitaine", Bordeaux, France.

\section{References :}

[1] Frenkel J. Viscous flow of crystalline bodies under the action of surface tension. J Appl Phys 1945; 9(5):385-91.

[2] Kuckzinsky GC. Study of the sintering of glass. J Appl Phys 1949;20:1160-3.

[3] Kingery WD, Berg M. Study of the initial stages of sintering solids by viscous flow, evaporationcondensation, and self-diffu-sion. J Appl Phys 1955;26(10): 1205-12.

[4] Mackenzie JK, Shuttleworth R. A phenomenological theory of sintering. Proc Phys Soc 1949;62:833-52.

[5] Coble RL. Sintering crystalline solids. I Intermediate and final diffusion models. II Experimental test of diffusion models in powder compacts. J Appl Phys 1961;32(5):787-99.

[6] Exner HE, Petzow G. Shrinkage and rearrangement during sintering of glass spheres. In: Sintering and catalysis. In: Kuckzinsky GC, editor. Materials Science Research, vol. 10. New York: Plenum Press; 1975. p. 279-93.

[7] Scherer GW. Sintering of low-density glasses: I, Theory of sintering. J Am Ceram Soc 
1977;60:236-9.

[8] Giess EA, Fletcher JP, Herron LW. I sothermal sintering of cordierite-type glass powders. J Am Ceram Soc 1984;67(8): 549-52.

[9] German RM. Sintering theory and practice. New York: Wiley-Interscience; 1996.

[10] Lange FF. Sinterability of agglomerated powders. J Am Ceram Soc 1984;67(1): 83-9.

[11] Zhao J, Harmer MP. Effect of pore distribution on microstructure development: Part I. J Am Ceram Soc 1988; 71(2): 113-20;

Part II. J Am Ceram Soc 1988; 71(7):530-9;

Part III. J Am Ceram Soc 1992; 75(4):830-43.

[12] Exner HE, Giess EA. A stereological-based equation for isotropic shrinkage during sintering by viscous flow. Science of sintering 7th. New York: Plenum Press; 1989. 73-85.

[13] Svoboda J, Riedel $\mathrm{H}$. Pore boundary interactions and evolution equations for the porosity and grain size during sintering. Acta Metall Mater 1992; 40:2829-40.

[14] Liu Y, Patterson BR. A stereological model of the degree of grain boundary-pore contact during sintering. Metal Trans 1993;24A: 1497-505.

[15] Scherer GW. Viscous sintering of a bimodal pore-size distribution. J Am Ceram Soc 1984; 67: 709- 15.

[16] Jagota A, Mikeska KR, Bordia RK. Isotropic constitutive model for sintering particle packing. J Am Ceram Soc 1990; 73(8): 2266-73.

[17] Baruchel J, Buffie 're JY, Maire E, Merle P, Peix G. X-ray tomography in material science. Paris: Herme's Sciences Publication; 2000. p. 204.

[18] Babout L, Maire E, Buffie` re JY, Fouge' res R. Characterization by X-ray computed tomography of decohesion, porosity growth and coalescence in model metal matrix composites. Acta Mater 2001; 49: 2055-63.

[19] Phillips DH, Lannutti JJ. X-ray computed tomography for the testing and evaluation of ceramic process. Am Ceram Soc Bull 1993; 72(11):69- 75.

[20] Deis TA, Lannutti JJ. X-ray computed tomography for evaluation of density gradient formation during the compaction of spray-dried granules. J Am Ceram Soc 1998;81(5): 1237-47.

[21] Lu P, Lannutti JJ, Klobes P, Meyer K. X-ray computed tomography and mercury porosimetry for evaluation of density evolution and porosity distribution. J Am Ceram Soc 2000; 83(3): 518- 22.

[22] Bernard D, Gendron D, Borde' re S, Heintz J M. First direct 3D visualisation of microstructural changes during sintering. ESRF Highlights 2001;2001: 102-3. Available from: http://www.esrf.fr/ info/science/highlights/2001/applied/APPL2.html.

[23] Chaminade JP, Viraphong O, Guillen F, Fouassier C, Czirr B. Crystal growth and optical properties of new neutron detectors $\mathrm{Ce}^{3+}: \mathrm{Li6R}(\mathrm{BO}) 3(\mathrm{R}=\mathrm{Gd}, \mathrm{Y})$. IEEE Trans Nucl Sci 2001;48(4): $1158-61$.

[24] Stanley JH. Computed tomography: the physical and mathematical basis of a powerful new industrial inspection technique. ASTM Standard News 1988:44-9.

[25] Censor Y. Finite serie-expansion reconstruction methods. Proc IEEE 1983; 71(3):409- 19.

[26] Herman GT. Image reconstruction from projections: the fundamentals of computerized tomography. New York: Academic Press; 1980.

[27] Natterer F. Numerical methods in tomography. Acta Num 1999; 8:107-41.

[28] D. Gendron, Numerical and experimental study of sintering at the grain scale. PhD. thesis, University Bordeaux I, 2001.

[29] Borde` re S, Bernard D, Gendron D, Heintz J M. Characterization of elementary sintering processes using Monte-Carlo simulation and X-ray computed microtomography. In: Mammoli AA, Brebbia CA, editors. Computational methods in materials characterisation. Southampton (UK): WIT Press; 2004. p. 23-32. 


\section{Figures:}
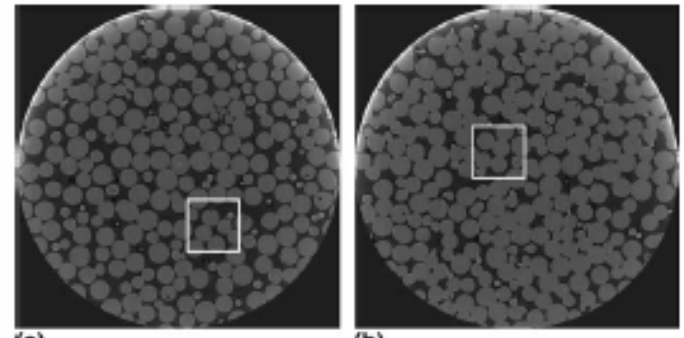

(a)

(b)
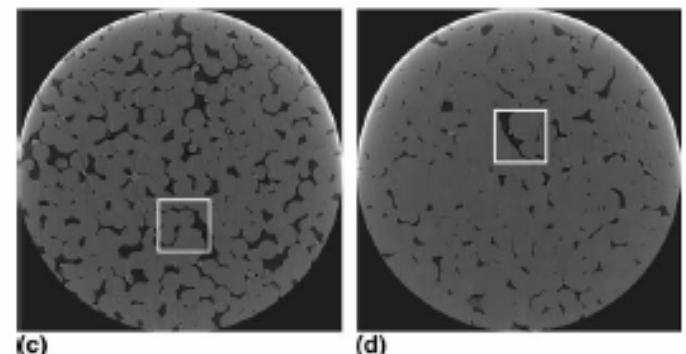

(d)

Fig. 1. Images of the same section of a glass powder sample for different sintering times, $t_{x}$ (a) 20 , (b) 120 , (c) 180 , (d) $270 \mathrm{~min}$. The squares indicate the area that has been reconstructed. 

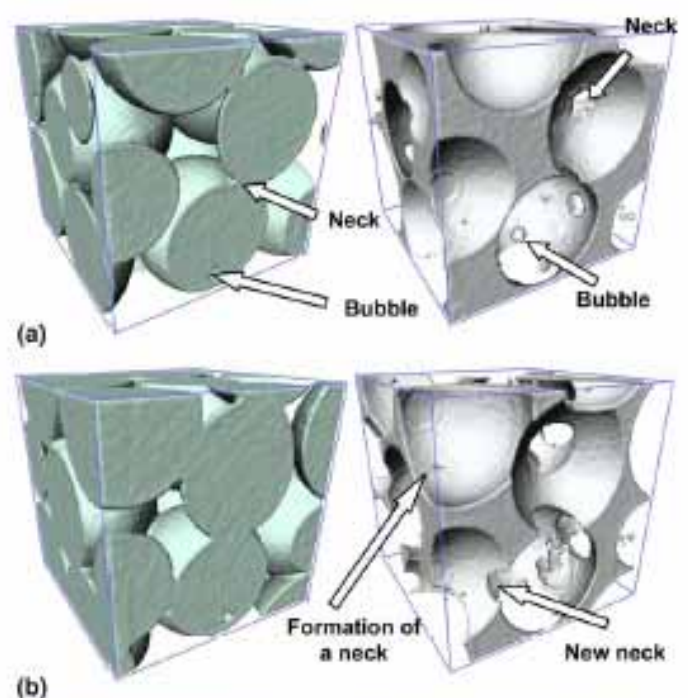

(b)

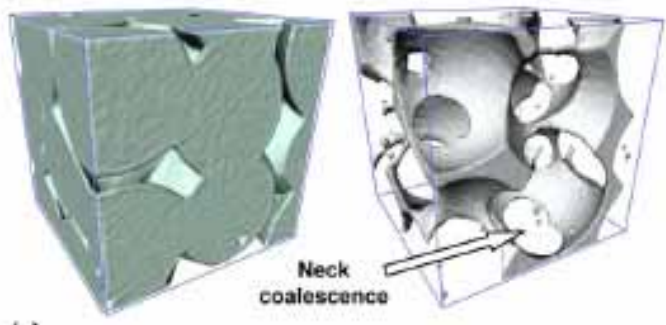

(c)

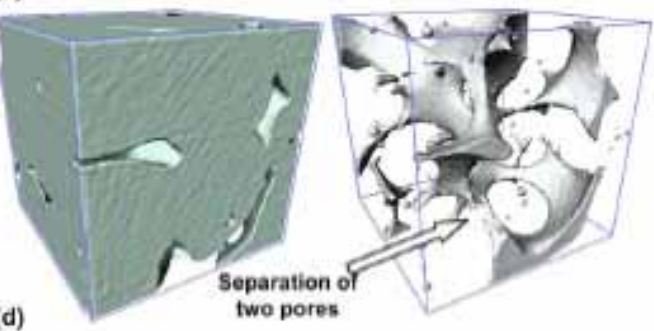

Fig. 2. Local morphological evolution of a glass powder sample (left. solid phase, right void space) as a function of sintering time, $t_{*}$ : (a) 20 , (b) 120 , (c) 180 , (d) $270 \mathrm{~min}$. 

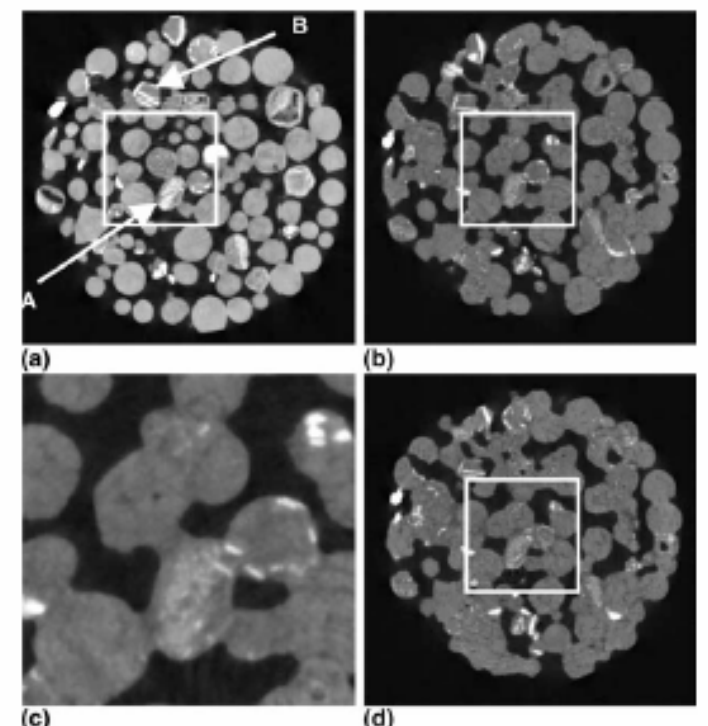

(d)

Fig. 3. Images of the same section of a lithium borate sample for different sintering times: (a) $t_{\mathrm{x}}=10 \mathrm{~min}$, (b) $t_{\mathrm{x}}=100$, (c) zoom on section (b), (d) $t_{\mathrm{x}}=190 \mathrm{~min}$. The squares indicate the area that has boen reconstneted. 

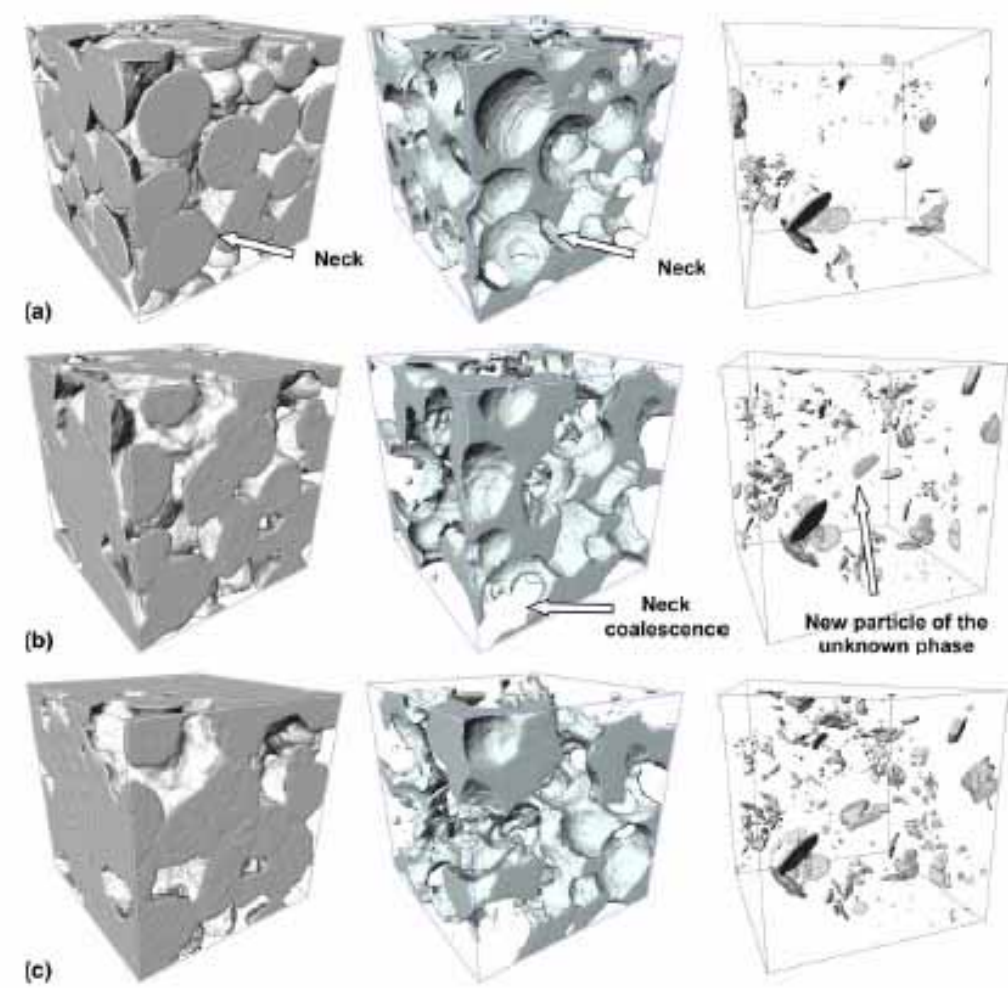

Fig. 4. Morphological evolution of a lithium borate sample sample (left: solid phase, ente: void space, right: unknown phase) as a funetion of sintering time, $t_{x}$ : (a) 10, (b) 100 , (c) 190 min. 


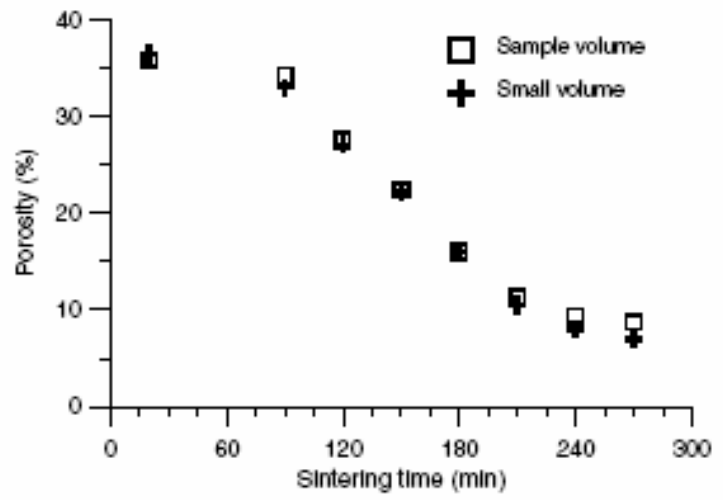

Fig. 5. Evolution of porosity during sintering for the whole glass powder sample and the small-reconstructed volume. The sibe of the symbol represents the error associated to the measurements.

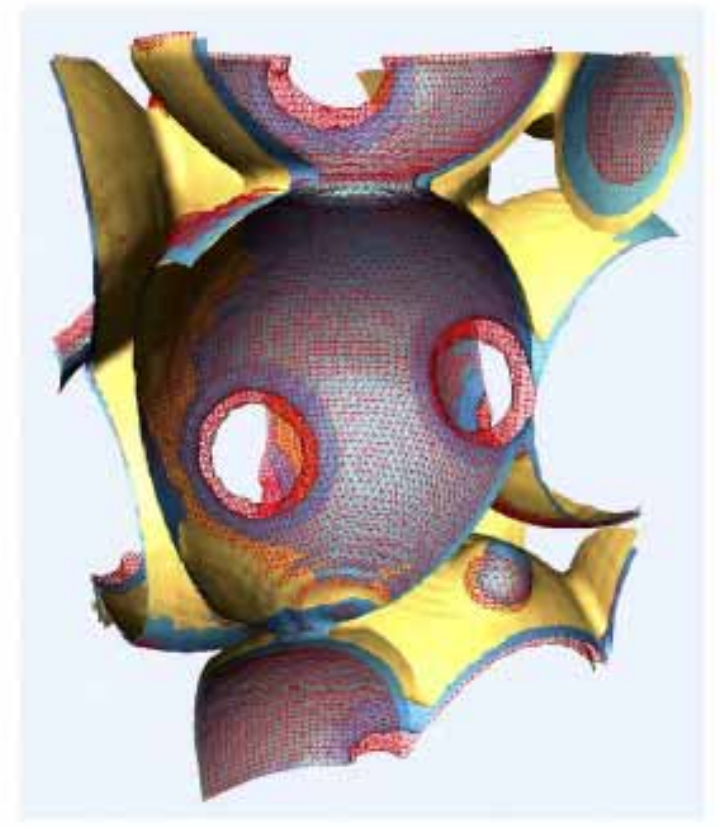

Fie 6. Visualisation of the same soda-line glass grain (diameter $\approx 120$ $\mu \mathrm{m}$ ) after different sintering times: Red lines correspond to $i_{2}=20 \mathrm{~min}$, transparent blue to $t_{x}=90 \mathrm{~min}$ and yellow to $t_{x}=120 \mathrm{~min}$. The good agreement between the surfaces proves that at the beginning of sintering and for this material, matter displacement is essentially bealised near the nocks. 


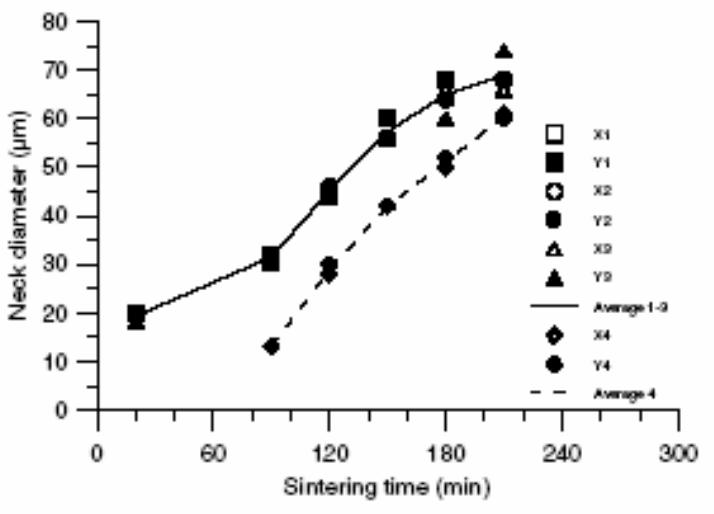

Fig. 7. Evolution of the neck size as a function of sintering time ( $X_{i}$ and $Y_{i}$ are two perpendicular diameters of the $i$ neck). 